\title{
Real-Time Iot Framework For Epileptic Seizures Detection And Alert System
}

\author{
Ms.S.Archana, ${ }^{2}$ K.Ammu, ${ }^{3}$ S.Bhuvaneshwari, ${ }^{4}$ S.Tamilmozhi, ${ }^{5}$ S.Vidhya \\ ${ }^{1}$ Assistant professor, ${ }^{2,3,4,5}$ Students \\ 1,2,3,4,5 Department of Biomedical Engineering \\ ${ }_{1,2,3,4,5}$ Adhiyamaan College Of Engineering, Hosur-635109, Tamilnadu, India.
}

\begin{abstract}
In this paper,it is a device that is designed for detecting occurrence of epileptic seizures in such cases, it is also a wearable which is smart detector and alert system, we can save the life of people who are really affected with the condition of epilepsy. It performs dual role such as detecting and sensing and se3nding alert system. To perform all the above process the main components used to detect are accelerometer, pressure sensor, heart beat sensor, GSM module to send an SMS and IOT framework.
\end{abstract}

Index Terms: Microcontroller, Sensors, IOT framework.

\section{INTRODUCTION}

Epilepsy is a neurological disorder which causes the sudden increase in brain activity, this causes disturbance in brain and cause seizures.Many people in this world suffers from epilepsy and needed treatment for their sufferings so we use IOT frame work to overcome their need for diagnosis .this device gains the bio signal from the body and transmits into a coded signal. The receiver gains the coded signal as decode signal and sends message to alert the doctors and guardians .hence the patient can be saved before the occurrence of epilepsy by using this gadget.

\section{OBJECTIVE}

This electronic gadget using IOT framework provides healthcare to all remote and advances the smart system in healthcare.The main aim of the project is to provide an IOT based electronic gadget for the epilepsy patients to protect themselves from the seizuresand heart attacks. This gadget gains the signals from human body hence this can avoid the sudden cause of epilepsy.

\section{LITERATURE OF SURVEY}

Patients that undergo treatment in the epilepsy aremonitored with different physical parameters, including audio. Which can have a large positive note on the daily care of epilepsy patients?This device has many stages that includes initially microphone array and noise subtraction and finally detected by audio classification so the features are gained by signals.the LCD display indicates that abnormal movement is detected. Here we use GPS to transfer the location and patients parameters to the concerned doctors and guardians.

\section{METHODOLOGY}

\section{A .Block Diagram}

Our system puts forward a smart system that uses Sensors to find patient health and uses internet to inform their doctors in case of any issues. Our system uses temperature as well as heartbeat sensor. The signal gained from the sensors is sent to a microcontroller which is in turn connected to an LCD display and Wi-Fi to provide alert system.

Changes detect3ed by the system such as increase in heart rate or temperature, the system alerts the user about the patients status over IOT and also shows details of heartbeat and temperature of patient live over the internet. Thus IOT based patient health tracking system completely uses interne to monitor patient health.

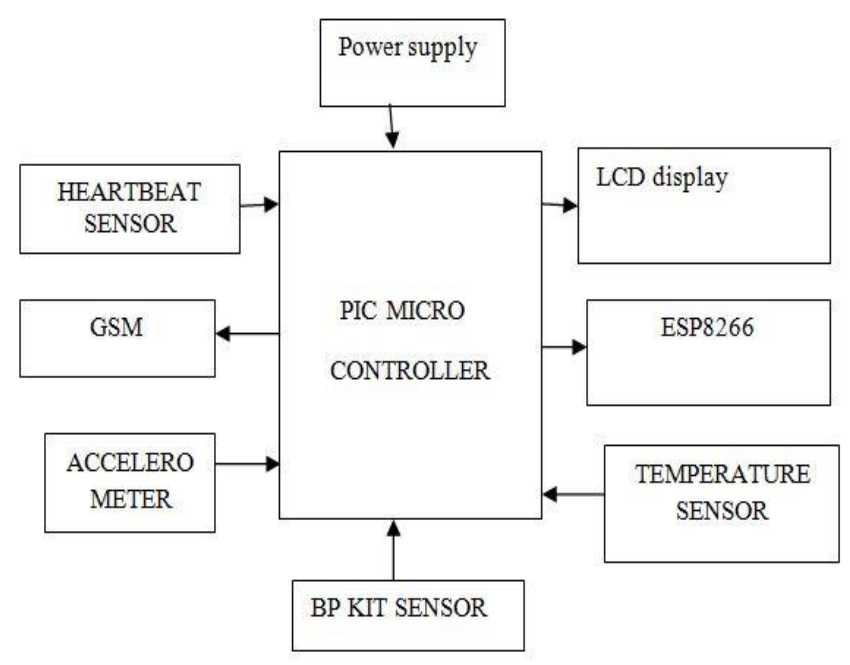

Fig 1. Block diagram

This system is based on pic microcontroller which is used to collect the patient data through sensors and informed through GSM module and uploaded to the server. In the system $\operatorname{lm} 35$ sensor used as temperature measurement of body which is real time temperature varies with different heat and blood pressure sensor used as 4 bit module, getting pressure value depend on body pressure and send to controller.

Accelerometer sensor is used as patient position condition which is differs and depending on body position. And the heartbeat sensor is used to measure the heart rate of patient. The ESP8266 module used to upload the data to server which is viewing in the thingspeak.com website. The IOT data monitoring was viewed by user login id and password. All the data displayed in LCD display. 


\section{Available online at www.ijrat.org}

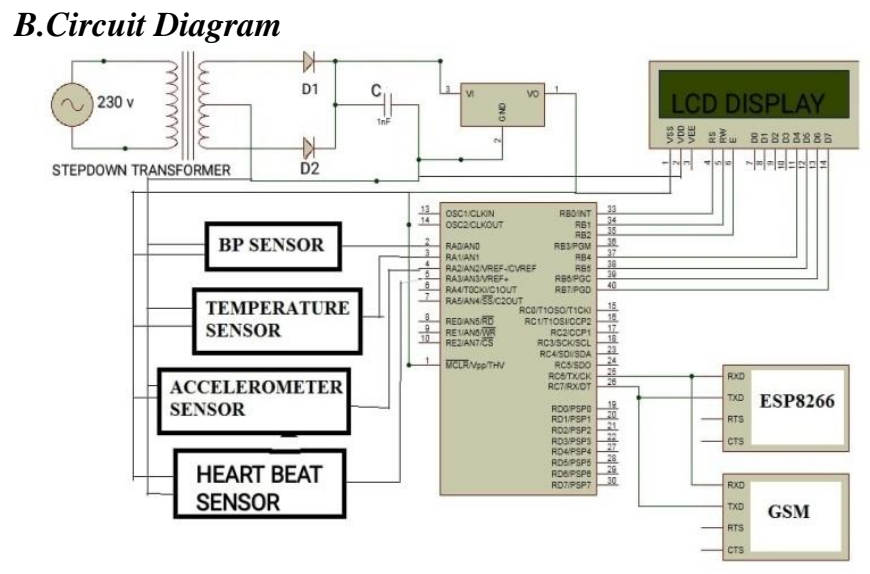

Fig.2Circuit diagram

Temperature Sensor and Accelorometer is a device used as a digital conversion.A pic microcontroller A0,A1 is an analog pin which is used to read the analog so the output of the temperature and accelorometer is given into the kit as well as LCD display needs a seven pins totally three which is used as a control pin and then the four pins used for data pins and used as a four bit mode.port B1,B2,B3 is connected to the control pin and then the port $\mathrm{B} 4, \mathrm{~B} 5, \mathrm{~B} 6, \mathrm{~B} 7$ is connected to the data pins.

ESP8266 is a IOT device which is used to send the data into cloud by the transmitter pin is connected to the receiver pin of the ESP8266.gsm model which is to be used to alert the signal to the concerned doctor or relatives about the patient emergency situation. It gets information from the controller and send sms to the relative person.we need a DC voltage for power supply so that use a step down from the input side and then convert AC into DC after converting voltage regulator to be used for getting a approximately 5V.Pressure sensor which is connected into D port.

\section{RESULT}

The patient affected by epilepsy will have sudden increase in heart rate and temperature hence this increases the movement and pressure in patient parameter. Initially when the patient is in normal condition the heart rate displayed in normal range that is 70 beats per minute and average temperature 30 is displayed. During the occurrence of epilepsy there raise the heart rate and temperature, so the LCD display indicates that abnormal movement is detected. Here we use GPS to transfer the location and patients parameters to the concerned doctors and guardians.

We have used IOT to interconnect the sensors and as the input output lines through the microcontroller.IOT is software we are using this through the laptop to show the results like the condition of the patient at the time of getting effected with the seizers and heart attacks. During epilepsy occurrence the symptoms and condition can be observed through the screen of laptop in the IOT module and a GPS module for alert systems and messages with patient parameters.

\section{CONCLUSION}

It is a weightless device, rugged, affordable wearable device which helps millions of people affected by epilepsy around the world. This system also incorporates GPS system so it's easily expandable and capture and transmits various parameters like ECG; body temperature etc. GPS system also helps in tracking the exact location of the patient during the occurrence of epilepsy.

\section{ACKNOWLEDGMENTS}

We thankful to Dr .T.S.UDHAYA SURIYA, M.E., Ph.D., Head of the Department of Biomedical Engineering, Adhiyamaan College of Engineering, for her most valuable guidance, advice and encouragement in all stages of our project.

We would like to extend our gratitude to Dr.T.S.UDHAYA SURIYA, M.E., Ph.D., Head of the Department of Biomedical Engineering, Adhiyamaan College of Engineering, for her valuable guidance and support to complete our project successfully.

\section{REFERENCES}

[1] O. Devinsky, "Sudden unexpected death in epilepsy," volume no 19, 2011.

[2] S. Beniczky, T. Polster, T. W. Kjaer, and H. Hjalgrim, "Detection of generalized tonicclonic seizures by a wireless wrist accelerometer" volume no 9,2013.

[3] J. F. Annegers and S. P. Coan, "SUDEP: Overview of definitions and review of incidence data," Seizure: European Journal of Epilepsy,vol.8,(6), pp. 347-352, 1999.

[4] K. V. Poppel, S. P. Fulton, A. McGregor, M. Ellis, A. Patters, and J. Wheless, "Prospective Study of the Emfit Movement Monitor, "J Child Neurol, vol. 00(0), pp.1-3,2013.

[5] G.deBruijne, P. Sommen, and R. Aarts, "Detection of epileptic seizures through audio classification," in $4^{\text {th }}$ European Congress of the International Federation for Medical and Biological Engineering(IFMBE),2008.

[6] K. Cuppens, B. Vanrumste, B. Ceulemans, L. Lagae, and S. VanHuf-fel, "Detection of epileptic seizures using video data," in Intelligent Environments (IE), 2010 Sixth International Conference on, 2010, pp.372373. 